\title{
UCRL-JRNL-222488
}

LAW RENCE LIVERMORE N A TIO N A L LABORATORY

\section{An Efficient Vector Finite Element Method for Nonlinear Electromagnetic Modeling}

A. C. Fisher, D. A. White, G. H. Rodrigue

June 29, 2006

Journal of Computational Physics 
This document was prepared as an account of work sponsored by an agency of the United States Government. Neither the United States Government nor the University of California nor any of their employees, makes any warranty, express or implied, or assumes any legal liability or responsibility for the accuracy, completeness, or usefulness of any information, apparatus, product, or process disclosed, or represents that its use would not infringe privately owned rights. Reference herein to any specific commercial product, process, or service by trade name, trademark, manufacturer, or otherwise, does not necessarily constitute or imply its endorsement, recommendation, or favoring by the United States Government or the University of California. The views and opinions of authors expressed herein do not necessarily state or reflect those of the United States Government or the University of California, and shall not be used for advertising or product endorsement purposes. 


\title{
An Efficient Vector Finite Element Method for Nonlinear Electromagnetic Modeling
}

\author{
A. Fisher ${ }^{a}$ D. White ${ }^{b}$ G. Rodrigue ${ }^{a}$ \\ aniversity of California, Davis \\ ${ }^{\mathrm{b}}$ Lawrence Livermore National Laboratory
}

\begin{abstract}
We have developed a mixed Vector Finite Element Method (VFEM) for Maxwell's equations with a nonlinear polarization term. The method allows for discretization of complicated geometries with arbitrary order representations of the $\mathbf{B}$ and $\mathbf{E}$ fields. In this paper we will describe the method and a series of optimizations that significantly reduce the computational cost. Additionally, a series of test simulations will be presented to validate the method. Finally, a nonlinear waveguide mode mixing example is presented and discussed.
\end{abstract}

\section{Introduction}

Continual growth in the understanding of nonlinear optics has introduced new possibilities in photonic device design. This growth has been fueled in part by the development of increasingly sophisticated analytical and numerical models. These models provide researchers with an understanding that enables them to design optical devices with increasing complexity and subtlty. Device engineers are beginning to examine devices with complicated geometries such as photonic crystals [1] and arrays of microring resonators [2]. Also, intricate nonlinear behaviors, such as supercontinuum generation [3], are being studied and considered for use in photonic devices.

Most optical modeling of nonlinear photonics employs the Slowly Varying Envelope Approximation (SVEA) to Maxwell's wave equation with a third order polarization response. This approach leads to the NonLinear Schrodinger Equation (NLSE), and this appraoch illuminates basic nonlinear optical effects such as the Kerr Effect, Four Wave Mixing, and Self Phase Modulation (SPM) [4]. Some work

Email addresses: fisher47@llnl.gov (A. Fisher), white37@llnl.gov (D. White), ghrodrigue@ucdavis. edu (G. Rodrigue). 
has been done to model nonlinear optical devices exibiting on-axis behavior using the Beam Propagation Method (BPM) with 2nd order indices of refraction [5]. These methods are computationally efficient, but do not represent a true solution to the full Maxwell's equations. Recently, a great deal of work has been done on modeling the full wave nonlinear Maxwell equations using a Finite Difference Time Domain (FDTD) approach [6][7]. These methods allow for full vectoral wave solutions but, but "staircasing" errors are introduced when applied to problems with curved geometry.

In this paper, we present a full wave 3D Vector Finite Element Method (VFEM) that can be used to model nonlinear photonics effects in complicated geometries. This method solves the coupled first-order Maxwell's equations with a third-order nonlinear polarization term with Debye relaxation. These equations are discretized in space using the Galerkin method with $H(\mathrm{curl})$-confoming "edge element" basis functions for the electric field and $H(d i v)$-conforming "face element" basis functions for the magnetic flux density. These 3D basis functions were first proposed in [8] and the properties of these basis functions have been investigated by many researchers [9] [10] [11] [12] [13] [14] [15] [16] [17]. For electrically large problems numerical dispersion is an issue [18] [19] [20] [21], and this can mitigated by using higher-order basis functions combined withhigher-order time integration [22] [23]. One dissadvantage of higher-order basis functions is that the finite element matrices become significanlty less sparse. While there is general agreement on the proper polynomial spaces underlying higher-order hexahedral $H(\mathrm{curl})$-confoming and $H($ div $)$-confoming basis functions, there is much latitude in the in the specific degrees of freedom, resulting in varius interpolarory, spectral, or heirarchical bases. By combining special numerical numerical quadratures with special interpolatory degrees of freedom, it is possible to develop a method that increases the sparsity of the matrices without degrading accuracy [24], and this approach is employed here.

Two specific issues with using a higher-order finite element method for the nonlinear Maxwell equations are investigated here. First, the time-discretization of the non-linear term is designed so that it does not require a non-linear Newton-type iteration at every time step, only a standard linear system solution involving a mass matrix is required. Second, since the permittivity and conductivity are time-varying, the various matrices that involve these quantities will need to be recomputed at every time step. Wereas a typical nonlinear mechanics code would simply recompute all matrices from scratch at every time step, since our computational mesh nodes do not move, computational optimizations are developed that bring memory and CPU usage down significantly. Finally, we present a series of validation simulations to demonstrate the accuracy of the new method, and use the method to examine nonlinear waveguides. 


\section{Mathematical Model}

Nonlinear electromagnetic phenomenon in a charge free domain is modeled by Maxwell's equations

$$
\begin{array}{r}
\nabla \cdot \mathbf{D}=0 \\
\nabla \cdot \mathbf{B}=0 \\
\dot{\mathbf{B}}=-\nabla \times \mathbf{E} \\
\dot{\mathbf{D}}=\nabla \times \mu^{-1} \mathbf{B}-\sigma \mathbf{E}-\mathbf{J}_{s} \\
\mathbf{D}=\varepsilon_{0} \mathbf{E}+\mathbf{P}(\mathbf{E})
\end{array}
$$

where the equations are, respectively, Coulomb's Law, the absence of magnetic monopoles, Faraday's Law, Ampere's Law, and electronic displacement [25]. We expand the polarization function in the usual power series for nonlinear optics [4].

$$
\mathbf{P}(\mathbf{E})=\varepsilon_{0}\left(\chi^{(1)} \mathbf{E}+\chi^{(2)} \mathbf{E}^{2}+\chi^{(3)} \mathbf{E}^{3}\right)
$$

We now restrict the model to isotropic materials so that the second order term is eliminated due to inversion symmetry, and the third order term to is simplified to $\chi^{(3)}(\mathbf{E} \cdot \mathbf{E}) \mathbf{E}$ with a scalar $\chi^{(3)}$. We can now express $\mathbf{D}$ from (1) as follows

$$
\begin{array}{r}
\mathbf{D}=\varepsilon_{e f f} \mathbf{E} \\
\varepsilon_{e f f}=\varepsilon_{0}\left(\chi^{L}+\chi^{N L}\right)
\end{array}
$$

where $\chi^{L}$ and $\chi^{N L}$ are the linear and nonlinear parts of $\varepsilon_{e f f}$. Representing $\mathbf{D}$ in this manner provides useful advantages. The nonlinearity is contained within a material response parameter that can be computed explicitly, and the splitting provides the flexibility to choose constitutive models separately for the linear and nonlinear portions of the permittivity. For our method we use the usual relative permittivity for the linear part and introduce a Debye time relaxation for the nonlinear part in order to model finite delay in the nonlinear response, specifically

$$
\begin{array}{r}
\chi^{L}=\varepsilon_{r} \\
\dot{\chi}^{N L}+\frac{1}{\tau} \chi^{N L}=\frac{1}{\tau} \chi^{(3)} \mathbf{E} \cdot \mathbf{E}
\end{array}
$$

where $\varepsilon_{r}$ is the usual relative permittivity and $\tau$ is the material nonlinear response time. Substituting (3) for $\mathbf{D}$ in (1) and carrying out the usual product rule for the time derivative we obtain the final form of Faraday's law used in our method.

$$
\varepsilon_{e f f} \dot{\mathbf{E}}+\dot{\varepsilon}_{e f f} \mathbf{E}=\nabla \times \mu^{-1} \mathbf{B}-\sigma \mathbf{E}-\mathbf{J}_{s}
$$

The $\varepsilon_{e f f} \dot{\mathbf{E}}$ term is the usual time derivative from Faraday's law with an effective permittivity while the $\dot{\varepsilon}_{e f f} \mathbf{E}$ term is a conductivity induced by changes in the non- 
linear response field.

\section{Vector Finite Element Method}

Given the properties of the different vector and scalar fields in (1), a mixed method, with the flexibility to represent each field in different a finite element space, is a natural choice. Following the approach in [26], we represent the fields in a discrete differential forms framework, whereby $\mathbf{E}$ fields are represented by 1 -forms, $\mathbf{B}$ fields by 2 -forms, and $\chi^{(N L)}$ fields by 3 -forms. Using this framework ensures that all of the necessary electromagnetic field properties are obeyed. For instance, the 1-form basis representations have tangential continuity, but can allow for normal discontinuities which are expected in $\mathbf{E}$ fields at material boundries. In addition, the differential forms framework provides a convenient notation to express the semi-discrete finite element equations.

Although hexahedral elements were chosen, none of the theory in the discrete differential framework limits the application to these elements. However, the computational optimizations that will be discussed later in this paper are best defined on a hexahedral geometry.

\subsection{Vector Basis Functions}

To begin we introduce the set of Lagrange interpolatory polynomials of degree $p$. These $p+1$ polynomials are defined by a distinct set of real valued interpolation points denoted by the symbol $X$ with $X=\left\{x_{0}, x_{1}, \ldots, x_{p}\right\}$. They are constructed so that the $i$ th polynomial has a value of 1 at $x_{i}$ and a value of 0 at all other interpolation points. This construction leads to a simple formula for the Lagrange interpolatory polynomials given by:

$$
L_{i}^{p}(x ; X)=\prod_{\substack{j=0 \\ j \neq i}}^{p} \frac{\left(x-x_{j}\right)}{\left(x_{i}-x_{j}\right)}
$$

By virtue of this construction, these polynomals satisfy the interpolation property given by

$$
L_{i}^{p}\left(x_{j} ; X\right)=0 ; \forall i \neq j
$$

Arbitrary order vector basis functions are constructed for all of the fields found in (1) using the Lagrange interpolatory polynomials within the discrete differential forms framework. For $\mathbf{E}$ and $\mathbf{J}_{s}, p$ order 1-form interpolatory vector basis functions 
are constructed as follows

$$
\begin{aligned}
& \psi_{i j k}^{1(x)}=L_{k}^{p-1}(x ; G) L_{i}^{p}(y ; B) L_{j}^{p}(z ; B) \hat{x} \\
& \psi_{i j k}^{1(y)}=L_{i}^{p}(x ; B) L_{k}^{p-1}(y ; G) L_{j}^{p}(z ; B) \hat{y} \\
& \psi_{i j k}^{1(z)}=L_{i}^{p}(x ; B) L_{j}^{p}(y ; B) L_{k}^{p-1}(z ; G) \hat{z} \\
& \text { for } i, j=0, \ldots, p ; k=0, \ldots, p-1
\end{aligned}
$$

where $G$ and $B$ represent the Gauss-Legendre and the Gauss-Lobatto quadrature points on the region $[0,1]$. These interpolation locations are carefully chosen to be useful for our computational optimizations. It should also be noted that these 1-form basis functions enforce tangential continuity when degrees of freedom are shared across adjacent element boundaries while leaving open the possibility of normal discontinuities. This allows for the accurate modeling of normal discontinuities in the $\mathbf{E}$ field across material boundries.

In a similar manner, $p$ order 2-form interpolatory vector basis functions are constructed to represent $\mathbf{B}$ as follows.

$$
\begin{aligned}
& \psi_{i j k}^{2(x)}=L_{k}^{p}(x ; B) L_{i}^{p-1}(y ; G) L_{j}^{p-1}(z ; G) \hat{x} \\
& \psi_{i j k}^{2(y)}=L_{i}^{p-1}(x ; G) L_{k}^{p}(y ; B) L_{j}^{p-1}(z ; G) \hat{y} \\
& \psi_{i j k}^{2(z)}=L_{i}^{p-1}(x ; G) L_{j}^{p-1}(y ; G) L_{k}^{p}(z ; B) \hat{z} \\
& \quad \text { for } i, j=0, \ldots, p-1 ; k=0, \ldots, p
\end{aligned}
$$

These 2-form basis functions enforce normal continuity when degrees of freedom are share across adjacent element boundaries, while leaving open the possibility for tangential discontinuities. This allows for the accurate modeling of tangential discontinuities in the $\mathbf{B}$ field across material boundries.

Finally, the following $p$ order 3-form interpolatory scalar basis functions are constructed to represent the time varying constituitive parameters $\chi^{(N L)}$ and $\varepsilon_{e f f}$.

$$
\begin{array}{r}
\psi_{i j k}^{3}=L_{i}^{p-1}(x ; G) L_{j}^{p-1}(y ; G) L_{k}^{p-1}(z ; G) \hat{x} \\
\text { for } i, j, k=0, \ldots, p-1
\end{array}
$$

These 3-form basis functions do not enforce any continuity since there are no degrees of freedom shared across element boundaries. This makes them an ideal choice for the material constituitive parameters since material boundaries are most often discontinous themselves. 


\subsection{Vector Finite Element Equations}

We follow the standard vector finite element approach and take inner products of three equations from (1), (5), and (4) with test functions taken from the vector basis functions previously discussed. In addition, each of the fields in (1) is represented with the basis functions outlined for them previously. This procedure leads to the following Galerkin form

$$
\begin{array}{r}
\left\langle\sum_{j} \dot{b}_{j} \phi_{j}^{2}, \phi_{i}^{2}\right\rangle=\left\langle\sum_{j} e_{j} \nabla \times \phi_{j}^{1}, \phi_{i}^{2}\right\rangle \\
\left\langle d_{e f f} \sum_{j} \dot{e}_{j} \phi_{j}^{1}+\dot{d}_{e f f} \sum_{j} e_{j} \phi_{j}^{1}, \phi_{i}^{1}\right\rangle=\left\langle\mu^{-1} \sum_{j} b_{j} \nabla \times \phi_{j}^{2}-\sigma \sum_{j} e_{j} \phi_{j}^{1}-\sum_{j} j_{j} \phi_{j}^{1}, \phi_{i}^{1}\right\rangle \\
\left\langle\sum_{j}\left(d_{e f f}\right)_{j} \phi_{j}^{3}, \phi_{i}^{3}\right\rangle=\left\langle\varepsilon_{j}+\varepsilon_{0} \sum_{j} r_{j} \phi_{j}^{3}, \phi_{i}^{3}\right\rangle \\
\left\langle\sum_{j} \dot{r}_{j} \phi_{j}^{3}+\tau^{-1} \sum_{j} r_{j} \phi_{j}^{3}, \phi_{i}^{3}\right\rangle=\left\langle\tau^{-1} \chi^{(3)}\left(\sum_{j} e_{j} \phi_{j}^{1}\right) \cdot\left(\sum_{j} e_{j} \phi_{j}^{1}\right), \phi_{i}^{3}\right\rangle
\end{array}
$$

where $b$ is the 2-form representation of $\mathbf{B}, e$ is the 1 -form representation of $\mathbf{E}, d_{\text {eff }}$ is the 3 -form representation of $\varepsilon_{e f f}$, and $r$ is the 3 -form representation of $\chi^{N L}$. With the application of integration by parts and some trivial simplifications, this leads to the semi-discrete matrix equations

$$
\begin{array}{r}
M^{(2)} \dot{b}=-D^{(12)} e \\
M_{d_{e f f}}^{(1)} \dot{e}+M_{\dot{d}_{e f f}}^{(1)} e=\left(D_{\mu^{-1}}^{(12)}\right)^{T} b-M_{\sigma}^{(1)} e-M^{(1)} j_{s} \\
d_{e f f}=\varepsilon+\varepsilon_{0} r \\
\dot{d}_{e f f}=\varepsilon_{0} \dot{r} \\
\dot{r}+\frac{r}{\tau}=\frac{\chi^{(3)}}{\tau} e \wedge^{(12)} M^{(2)^{-1}} H^{(12)} e
\end{array}
$$

The matrices in these equations have the following form

$$
\begin{array}{r}
\left\{M_{\alpha}^{(1)}\right\}_{i j}=\int_{\Omega} \alpha \psi_{i}^{1} \cdot \psi_{j}^{1} d \Omega \\
\left\{M_{\alpha}^{(2)}\right\}_{i j}=\int_{\Omega} \alpha \psi_{i}^{2} \cdot \psi_{j}^{2} d \Omega \\
\left\{D_{\alpha}^{(12)}\right\}_{i j}=\int_{\Omega} \alpha\left(\nabla \times \psi_{i}^{1}\right) \cdot \psi_{j}^{2} d \Omega \\
\left\{H_{\alpha}^{(12)}\right\}_{i j}=\int_{\Omega} \alpha \psi_{i}^{1} \cdot \psi_{j}^{2} d \Omega
\end{array}
$$

where $M^{(1)}$ is a 1 -form Mass matrix, $M^{(2)}$ is a 2-form Mass matrix, $D^{(12)}$ is a Derivative matrix, and $H^{(12)}$ is a Hodge matrix. The mass matrices are well known in the finite element community, however the complexity of the other two requires some explanation. The Derivative matrix is a discrete representation of the curl operation and transforms a 1-form with material parameter to a 2-form. The Hodge 
matrix is a simple 1-form to 2-form mapping and can be used to transform from one form to the other using $M^{(2)} v^{(2)}=H^{(12)} v^{(1)}$. It should be noted that the $i$ and $j$ indices are taken from the proper degrees of freedom within the finite element mesh. Hence, they are different indices for each matrix. For example the $i$ 's in the Derivative matrix are taken from the 1-form degrees of freedom in the mesh while the $j$ 's are taken from the 2-form degreees of freedom. Since there are different numbers of 1-form and 2-form degrees of freedom in a mesh, the Derivative and Hodge matrices are rectangular.

Another matter of importance is the time dependence of $d_{e f f}$, and with it, the time dependence of $M_{d_{e f f}}^{(1)}$ and $M_{\dot{d}_{e f f}}^{(1)}$. Since this parameter changes with time, these mass matrices must be updated at every time step.

\subsection{Time Discretization}

In order to complete the description of method a time integration scheme is required. For low orders of accuracy, a simple leap-frog technique will suffice. However, in keeping with the spirit of arbitrary order calculations, an arbitrary order symplectic time integration scheme is applied. Symplectic time integration schemes are not new, nor is their application to Maxwell's equations [22], however there is the complication of the nonlinear response equations. In the scheme these equations are updated at the beginning of each time integration step with the same time step parameters as $e$. This approach leads to Algorithm 1, where the $\alpha$ 's and $\beta$ 's are the symplectic integration coeffecients found in Table 3.3. 


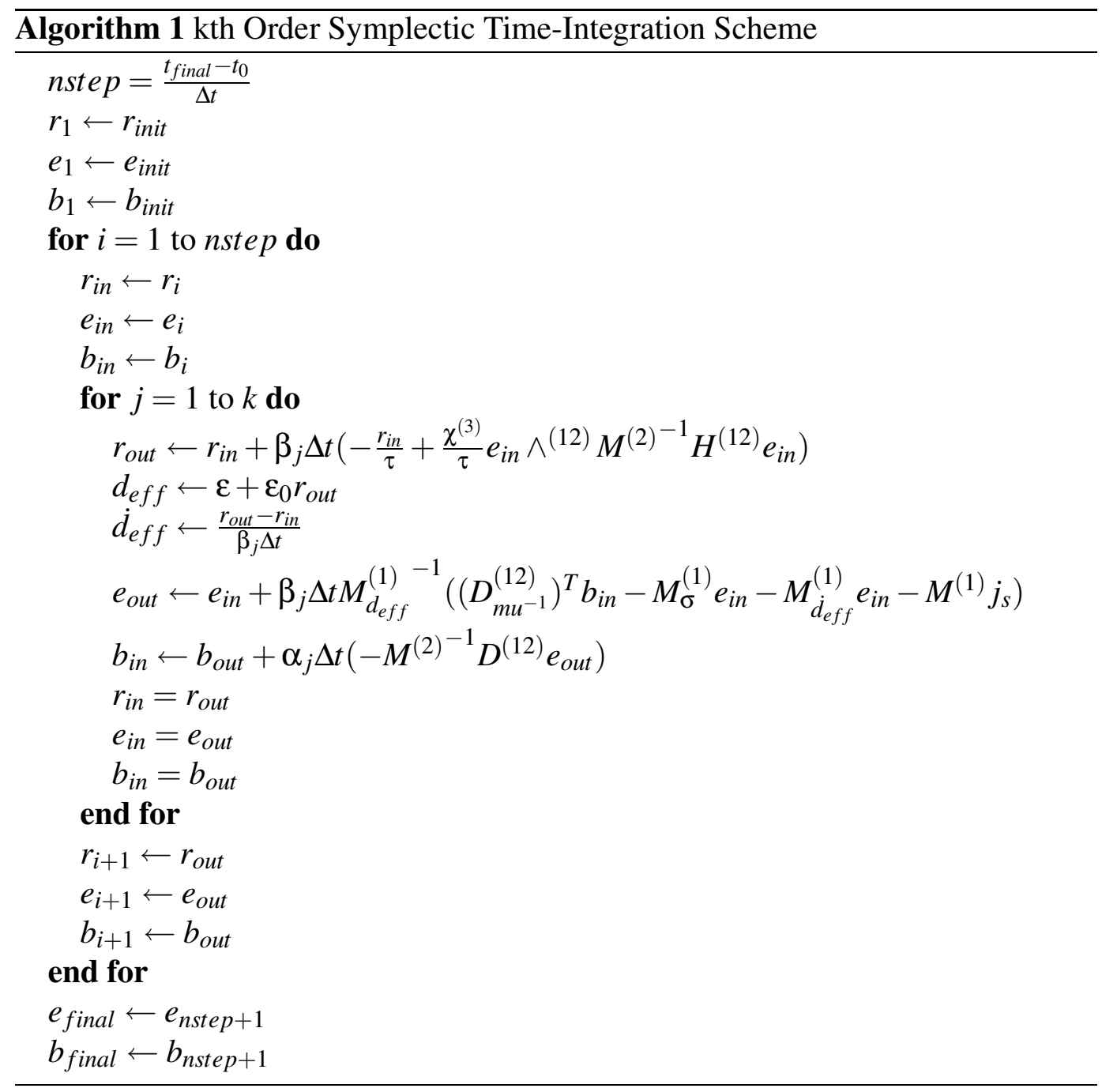




\begin{tabular}{cc}
\hline \multicolumn{1}{c}{ Order 1 } & \\
\hline$\alpha_{1}=1$ & $\beta_{1}=1$ \\
\hline Order 2 & \\
\hline$\alpha_{1}=1 / 2$ & $\beta_{1}=0$ \\
$\alpha_{2}=1 / 2$ & $\beta_{2}=1$ \\
\hline Order 3 & $\beta_{1}=7 / 24$ \\
$\alpha_{1}=2 / 3$ & $\beta_{2}=3 / 4$ \\
$\alpha_{2}=-2 / 3$ & $\beta_{3}=-1 / 24$ \\
\hline$\alpha_{3}=1$ & \\
\hline Order 4 & $\beta_{1}=0$ \\
$\alpha_{1}=\left(2+2^{1 / 3}+2^{-1 / 3}\right) / 6$ & $\beta_{2}=1 /\left(2-2^{1 / 3}\right)$ \\
$\alpha_{2}=\left(1-2^{1 / 3}-2^{-1 / 3}\right) / 6$ \\
$\alpha_{3}=\left(1-2^{1 / 3}-2^{-1 / 3}\right) / 6$ & $\beta_{3}=1 /\left(1-2^{2 / 3}\right)$ \\
$\alpha_{4}=\left(2+2^{1 / 3}+2^{-1 / 3}\right) / 6$ & $\beta_{4}=1 /\left(2-2^{1 / 3}\right)$ \\
\hline
\end{tabular}

Table 1

Symplectic Integration Coeffecients for Algorithm 1

All that remains for the description of the method is to define a stable time step $\Delta t$. The usual Courant-Friedrichs-Lewy (CFL) like stability condition for linear electromagnetics methods would be a good starting point for this nonlinear method. Unfortunately, the nonlinear method is occasionally unstable at such timesteps, which is not surprising given that similar problems have arisen in nonlinear FDTD methods [7]. However, simply setting the time step to $50 \%$ of the linear stability condition maintained stability for all of the nonlinear simulations we ran. Thus the CFL like stability condition for the symplectic time integration of our nonlinear VFEM equations is

$$
\Delta t \leq \frac{1}{\sqrt{\rho\left(\alpha_{i} \beta_{i} M^{(2)^{-1}} D^{(12)} M_{\varepsilon}^{(1)^{-1}} D_{\mu^{-1}}^{(12)^{T}}\right)}} ; \quad \forall i
$$

where $\rho$ denotes the spectral radius function and $M_{\varepsilon}^{(1)}$ is the usual 1-form mass matrix without the nonlinear permittivity variation. 


\section{Computational Considerations}

Each of the $k$ sub-steps of the higher order time step in Algorithm 1 requires 3 linear system solves. In addition, the $M_{\mathrm{d}_{e f f}}^{(1)}$ and $M_{\dot{d}_{e f f}}^{(1)}$ are dynamic, adding 2 matrix formation operations to each sub-step. Finally, the memory requirements of this method are high considering that there are up to 8 matrices required to complete an iteration.

First, the requirement of 3 linear solves per sub-step will be addressed. Within the framework of discrete differential forms, we can write $D^{(12)}=M^{(2)} T^{(12)}$ where $T^{(12)}$ is the Topological Derivative Matrix. This allows us to drop the matrix solve for the update of $b_{\text {out }}$ since the 2-form mass matrices cancel leaving only $T^{(12)}$. This matrix can be computed explicitly with the following projection

$$
T_{i j}^{(12)}=\operatorname{proj}_{\phi_{i}^{2}}\left\{\nabla \times \phi_{j}^{1}\right\}
$$

which depends only on the topological information in the mesh.

In addition, the linear solve in the update of $r_{\text {out }}$ can be dropped if we briefly step away from the framework of discrete differential forms. Looking back at the mathematical model (1), the right hand side of the nonlinear response model involves an $\mathbf{E} \cdot \mathbf{E}$ that must be represented by a 3-form. This can be done without the need for a linear solve using the following discretization.

$$
\mathbf{E} \cdot \mathbf{E} \approx \operatorname{proj}_{\phi_{i}^{3}}\left\{\left(\sum_{j} e_{j} \psi_{j}^{1}\right) \cdot\left(\sum_{j} e_{j} \psi_{j}^{1}\right)\right\}
$$

In practice this involves using the 1-form basis expansion to compute the $\mathbf{E}$ field vectors at the 3-form interpolation locations and setting the 3-form degrees of freedom with the $\mathbf{E} \cdot \mathbf{E}$ values obtained from those vectors. This approach eliminates the linear solve needed for the wedge product.

The final linear system solve cannot be eliminated in all cases. However, previous work on a lumping scheme for 1-form mass matrices can be used to reduce the computational cost of this solve by an order of magnitude on an arbitrary mass matrix, and eliminate the solve entirely on a mass matrix formed with an orthogonal mesh. The basic approach of this method is to use a set of 4 carefully constructed quadrature rules to approximate the 1-form mass matrix integral. In doing so, the mass matrix has far fewer non-zero entries so that sparse matrix multiplication requires fewer operations. Details can be found in [24].

Finally, the cost of the complete assembly of 2 mass matrices at each time step can be reduced considerably. In particular, the work on finite element matrix decomposition in [27] turns out to be quite valuable. Using these ideas, all of the finite 
element matrices in the method are decomposed in the following manner

$$
A_{m}^{(p q)}=Q^{T}\left(\sum_{k} M_{k} G_{k}\right) P
$$

where $P$ and $Q$ contain all of the topological information in the finite element mesh, $M_{i}$ is a diagonal matrix containing all of the material parameter information needed for the matrix at a particular, and $G_{i}$ is a block diagonal matrix containing all of the geometry information in the mesh. The sum runs across the $k 3$-form interpolation locations used to store material information on each element. These matrices can be constructed as follows

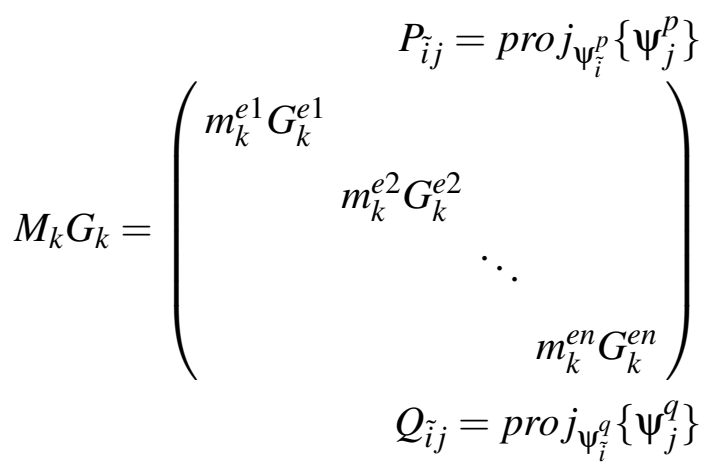

where the $\tilde{i}$ runs acress the elemental degrees of freedom the and the $j$ runs across the global degrees of freedom. The $m_{k}^{e a}$ values are $k$ th 3 -form degrees of freedom for the ath element. These values are exactly the values found in the 3-form representation of the material parameter. The $G_{k}^{e a}$ matrices are computed by using the bilinear forms found in (13) with the $k$ th 3 -form basis in place of the material on the $a$ th element. For example the $G$ matrices for the 1-form mass matrix would wave the following form

$$
\left\{G_{k}^{e a}\right\}_{\tilde{i} \tilde{j}}=\int_{\Omega} \psi_{k}^{3} \psi_{\tilde{i}}^{1} \cdot \psi_{\tilde{j}}^{1} d \Omega
$$

where the $\tilde{i}$ and $\tilde{j}$ run across the 1 -form degrees of freedom for element $a$ and $k$ runs across the 3-form degrees of freedom for element $a$.

In order to better understand how this decomposition works, it is worthwhile examine the action of each matrix in a matrix vector mutiply $A_{m}^{(p q)} x$. The multiplication by the $P$ matrix is a matrix takes the $p$-form $x$ vector and expands it into a nonassembled elemental version of $x$. The $G_{k}$ matrix takes the non-assembled $p$-form vector and translates it to a non-assembled $q$-form vector with all of the geometry and derivative information that was found in $A^{(p q)}$. The $M_{k}$ matrix simply applies the material information to the vector. Finally, multiplication by $Q^{T}$ takes the result in non-assembled form and assembles it into the final $q$-form vector.

The upside of this process is that the time varying material parameters are now removed from the rest of the matrix so that they can be updated without recomputing the entire matrix. However, one might argue that there are now more matrices 
to store and they are larger than than the standard matrices since they work with non-assembled degrees of freedom. However, memory costs for this approach can actually be reduced to levels below the requirements for the standard approach in many cases by relatively simple optimizations. First, since the $P$ and $Q$ matrices only contain topology information for the mesh and a particular $p$ or $q$ form, these matrices can be shared for all of the matrix decompositions across the entire method. Thus only 3 of these matrices must be stored, one for each of the three forms that are used in the method. Additionally, for many meshes, the $G$ matrix can undergo significant compression. Since the $G$ matrix only contains information about the geometry of the mesh and the derivative information that is uniform across the problem, any number of elements with the same geometry can be represented with a single elemental matrix. Many meshes have symmetries that allow this simple form of compression to significantly reduce the memory required for the $G$ matrix. In the optimal case of a cartesian mesh, the $G$ matrix can be represented by a single elemental matrix.

\section{Validation}

There is a great need for a rigorous program of validation with the proposal of a new computational method of this level of complexity. Validation is not only needed to ensure that the method is consistent with well accepted physical theory but also to determine the levels of error and rates of convergence that are expected when using the method at various orders of spatial and temporal accuracy.

Some unique complications arise in the case of nonlinear optical modeling. While there is an abundance of analytical work in the field, most of it provides solutions to the NLSE by using the SVEA. This makes it difficult to consider precise comparisons, such as $L_{2}$ error norms, between analytical and computational results since they are actually solving different equations. Any direct error measurements would need to decouple the error introduced by the computational method from the error introduced by solving different equations.

Fortunately, by setting the nonlinear parameter $\chi^{(3)}$ to 0 the computational method reduces to a linear vector finite element method for electromagnetic simulation that has already been through a great deal of validation and comparison to linear analytical results [28]. Given those results it is only necessary to test the physical effects that are introduced by the nonlinear parameter. 


\subsection{Analysis of the Nonlinear Response Variation}

In order to obtain a better understanding of the resolution needed to approximate $\chi^{N L}$ we perform an analytical analysis of the nonlinear response to simple sinusodial oscillations. We begin with the solution to nonlinear response ODE which is found easily using an integrating factor.

$$
\chi^{N L}=e^{-\frac{t}{\tau}} \int_{0}^{t} e^{\frac{t^{\prime}}{\tau}} \frac{\chi^{(3)}}{\tau} \mathbf{E} \cdot \mathbf{E} d t^{\prime}
$$

Considering only the time variation of an infinite plane wave we have an electric field of the form

$$
\mathbf{E}=E_{0} \cos \omega t \hat{\mathbf{y}}
$$

where $\omega$ is the radial frequency of the plane wave variations. Using this electric field function and assuming we are sufficiently far from the initial disturbance of turning the field on, we have the following approximation for the nonlinear response.

$$
\chi^{N L} \approx \chi^{(3)} E_{0}^{2} \frac{2 \tau \omega \sin 2 \omega t \cos 2 \omega t+4 \tau^{2} \omega^{2}+1}{8 \tau^{2} \omega^{2}+2}
$$

By substituting maximum and minimum values of 1 and -1 for both the sine and the cosine an upper bound for the range of the variation can be found. An integral over a period of the waves reveals the average value of the nonlinear response allowing for a bound for the normalized range of

$$
\frac{\Delta \chi^{N L}}{\chi^{\bar{N} L}}<\frac{8 \pi \alpha+2}{16 \pi^{2} \alpha^{2}+1}
$$

where $\alpha=\tau / T$ which is the response time of the material normailzed by the period of the wave. This tells us that as long as the nonlinear response time is greater than 1 period of the electric field wave, the fast sinusodial variations change $\chi^{N L}$ by less than $\pm 8.5 \%$ (see Figure 1). Response times for materials vary from $10^{-16} s$ to $10^{-9} s$ which corresponds to $0.03 T$ to $300,000 T$ for a wavelength of $1 \mu \mathrm{m}$. So all but the fastest responses can be accurately modeled with the mixed $2 \mathrm{nd} / 1 \mathrm{st}$ order method. Additionally, the magnitude of the nonlinear response is typically small compared to the linear response which makes the range of these sinusodial variations even smaller compared to the value of the effective permittivity $\varepsilon_{e f f}$. With such small variations it is reasonable to spatially subsample the fast sinusodial variations in

$\chi^{N L}$ and focus on having enough resolution to sample the variation in the magnitude of the wave over longer distances.

\subsection{D Numerical Dispersion}

Numerical dispersion is regarded as the dominant source of error in wave modeling simulations, and any method used to simulate such waves must go through 


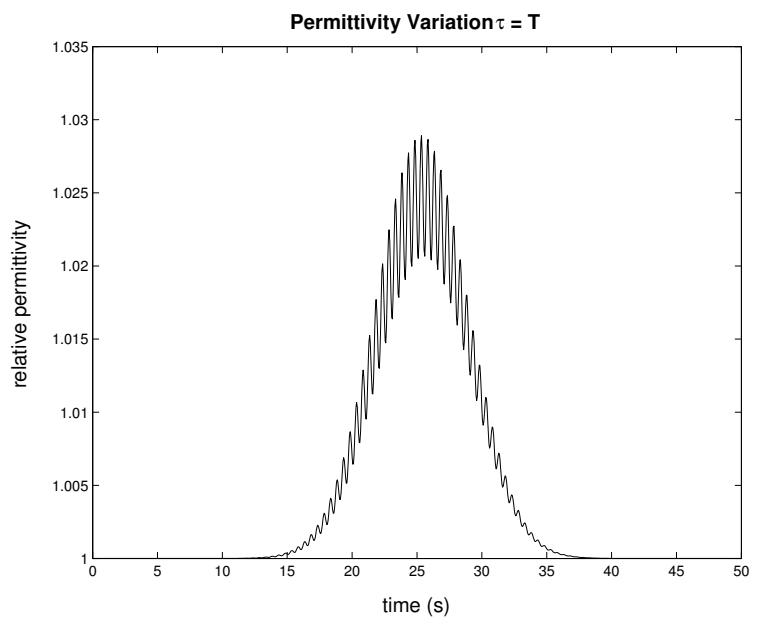

Fig. 1. Variation in relative permittivity in response to a sinusodially varying pulse with a gaussian envelope. $\tau$ is 1 period of the sinusodial variation and the nonlinear response is varying by roughly $\pm 8.5 \%$.

some form of dispersion analysis. Unfortunately, the complications added by the nonlinear parameter and the time varying mass matrix make a classic numerical dispersion analysis, such as the one found in [19], infeasible.

However, with the importance of numerical dispersion error in mind, we used another approach to measure the dispersive effects. A $1 \mu m \times 1 \mu m \times 100 \mu m$ rectangular prism was discretized by a $1 \times 1 \times 20001$ st order element mesh and a $1 \times 1 \times 1000$ 1 st order element mesh. Perfect Electric Conductor (PEC) boundary conditions were applied to the top and bottom $x z$ planes, while Neumann boundary conditions were applied to the $y z$ sides. Sinusodial time varying voltage sources with vectors in the $+y$ direction were applied to the $z=0 \mu m$ ends, while Neumann boundary conditions were applied to the $z=100 \mu m$ ends. This had the effect of injecting plane waves into a configuration that only allows variation in the $+z$ direction, effectively creating 1 dimensional simulations. On the $1 \times 1 \times 2000$ element mesh first order basis functions were used for all the fields, and on the $1 \times 1 \times 1000$ element mesh 2nd order basis functions were used for the $\mathbf{E}$ and $\mathbf{B}$ fields while 1st order basis functions were used for the $\chi^{N L}$ field.

Plane waves with wavelengths varying from 1.0 $\mu \mathrm{m}$ to $2.0 \mu \mathrm{m}$ and $\mathbf{E}$ field magnitudes varying from $1 \mathrm{~V} / \mathrm{m}$ to $1.5 \times 10^{8} \mathrm{~V} / \mathrm{m}$ were injected into a material with $\mu=\mu_{0}$, $\varepsilon=\varepsilon_{0}, \chi^{(3)}=2 \times 10^{-18}$, and $\tau=2 T$ where $T$ is the period of the injected wave. The propagation of these plane waves was simulated through 12000 time steps of $\Delta t=2.5 \times 10^{-17} \mathrm{~s}$, and snapshots were taken to measure the wave velocities.

The simplicity of this nonlinear electromagentics problem allows a very reasonable analytical approximation for the wave velocity to be found. If we consider a time- 

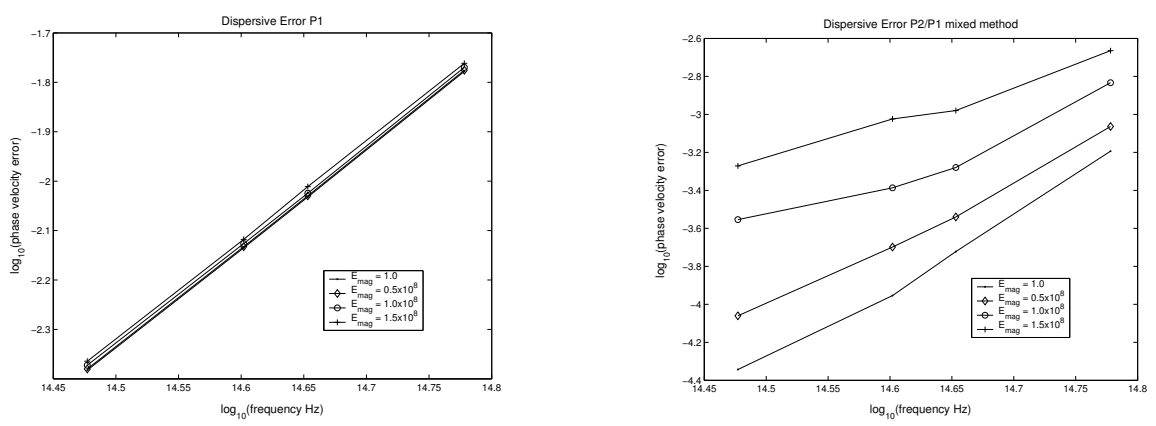

Fig. 2. Relative nonlinear phase velocity error as a function of frequency for 1st order elements and for the mixed 2nd/1st order method. Physical behavior is essentially linear with $E_{m a g}=1 \mathrm{~V} / \mathrm{m}$, however the nonlinearity perturbs $\varepsilon_{\text {eff }}$ by $4.5 \%$ with $E_{\text {mag }}=1.5 \times 10^{8} \mathrm{~V} / \mathrm{m}$.

averaged velocity, over a period of the wave, we can derive the following equation:

$$
<v>\approx \frac{1}{\sqrt{<\varepsilon_{e f f}>\mu}}=\frac{1}{\sqrt{\varepsilon_{0}\left(1+\chi^{(1)}+\frac{1}{2} \chi^{(3)} E_{m a g}^{2}\right) \mu}}
$$

This approximation is derived by assuming that the response time model acts as a flat, unweighted average over an optical period. This is of course not the case with the Debye relaxation model being used. However, it is a good approximation since the error will tend to overestimate $v$ half the time and underestimate $v$ the other half the time, with velocity measurements taken over many optical periods. One effect that is not captured effectively by that is not captured by this simple mode is the $\chi^{(3)}$ induced conductivity that arises from the $\dot{\varepsilon}_{e f f} \mathbf{E}$ term in (5). This induced conductivity causes some dampening of the plane waves which affects their magnitude and consequently their phase velocity. To correct for this effect, average magnitudes were measured from the simulated plane waves and used in the model for $E_{m a g}$ rather than initial values used for the simulation.

To complete the analysis of dispersion, error values (see Figure 2) were obtained by computing the differences between simulated wave velocities and the velocities given by the analytical model in (24). The method's order of accuracy can be found by fitting lines to the data and calculating their slopes. This procedure reveals that in the linear case, when $E_{m a g}=1$, the order of accuracy is for 1st order basis functions is 2.01 which is near the theoretical limit of 2 expected for linear methods with 1st order elements [21]. In the significantly nonlinear case when $E_{\text {mag }}=1.5 \times 10^{8} \mathrm{~V} / \mathrm{m}$, the order of accuracy is maintained at 2.00. This indicates that the 1st order discretization at these resolutions and magnitudes has dispersion errors that are still dominated by the usual discretization inaccuracies, and not the addition of the nonlinear response. When using the 2nd/1st order mixed scheme on the $1 \times 1 \times 1000$ element mesh, the results were more varied. In the linear limit with $E_{m a g}=1$ the order was 3.84 which is near the theoretical limit of 4 . As $E_{\text {mag }}$ is raised to $0.5 \times 10^{8} \mathrm{~V} / \mathrm{m}, 1.0 \times 10^{8} \mathrm{~V} / \mathrm{m}$, and $1.5 \times 10^{8} \mathrm{~V} / \mathrm{m}$, the order of accuracy falls to $3.31,2.39$, and 1.98 respectively. That with a large nonlinearity the $2 \mathrm{nd} / 1 \mathrm{st}$ order mixed scheme degenerates to 2 nd order accuracy, however the behavior with 
smaller nonlinearities approaches 4 th order accuracy.

Comparing the plots in Figure 2 shows that even in the case of large nonlinearity with $E_{\text {mag }}=1.5 \times 10^{8} \mathrm{~V} / \mathrm{m}$ there is roughly a factor of 10 improvement in dispersion error when using the $2 \mathrm{nd} / 1$ st order mixed method. This improvement comes at a significant increase in computational cost, however, the factor of 2 coarsening in the mesh ameliorates that increase. In a full 3 dimensional problem that coarsening reduces the number of elements by a factor of 8 while the increase to 2nd order basis functions for $\mathbf{E}$ requires that the 1-form elemental mass matrices are $54 \times 54$ with 702 non-zeros instead of $12 \times 12$ with 60 non-zeros for 1 st order (see [24] for number of non-zero calculations). Since a matrix equation solve involving the 1-form mass matrix must be performed at every time step the bulk of the work in the method comes from multiplying all of the element mass matrices by appropriate vectors at each step in the iterative solve. Given that sparse matrices are used to represent the element mass matrices, the number of multiplictaions that must be perfomed scales with the number of matrices and the number of non-zeros per matrix. This leads to an estimate of $1.46 \times$ the amount of work required for the $10 \times$ increase in accuracy gained by using the $2 \mathrm{nd} / 1$ st order mixed method. For comparison, to gain a $10 \times$ increase in accuracy using the 1 st order method alone would require a refinement factor of 3.16 leading to a factor of $31.55 \times$ increase in the number of elements in 3 dimensions and a $31.55 \times$ increase in computational cost.

\section{$5.32 D$ Self Focusing}

Self focusing is an optical behavior in which the nonlinear dielectric response causes a Gaussian beam to bend inward and focus while traveling through bulk material. This process is caused by the alteration of the local index of refraction at the beam due to the Kerr effect. This effect is intensity dependent and given by

$$
\begin{array}{r}
n_{e f f}=n_{0}+n_{2} I \\
n_{2}=\frac{1}{2 n_{0}} \chi^{(3)}
\end{array}
$$

where $n_{0}$ is the usual index of refraction and $n_{2}$ is the Kerr index. This variation causes parts of the beam at higher intensities to travel slower than parts of the beam at lower intensities, effectively introducing a distributed Gaussian lens. Usually the lensing effect is more than offset by beam divergence due to diffraction. However, if the beam intensity is high enough, the lensing effect overcomes the divergence and the beam self focuses.

While, to date, there are no exact solutions to Maxwell's equations for this problem, there are reasonable approximations that capture the flavor of the effect. Early work on the abberationless approximation made the assumption that the Gaussian Beam 
remains Gaussian throughout the self focusing process [29]. This approximation made it possible to calculate the critical beam power at which the beam neither focuses nor diverges. Later the effect of the abberations was added to refine the critical power predictions, yielding the well known Kelly formula [30]

$$
\begin{aligned}
& P_{c r}=\frac{(1.22 \lambda)^{2} v}{256 n_{2}} \\
& E_{c r}=\sqrt{\frac{16 P_{c r}}{v w_{0}^{2}}}
\end{aligned}
$$

where $v$ is the beam velocity in the medium, and $\lambda$ is the beam wavelength in the medium, and $w_{0}$ is the beam waist.

We used the method to simulate $2 \mathrm{D}$ Gaussian beams of various magnitudes. A $30 \mu m \times 1 \mu m \times 80 \mu m$ rectangular domain was discretized by $300 \times 1 \times 16001 \mathrm{st}$ order elements. PEC boundary conditions were applied to top and bottom, while Neumann boundary conditions were applied to the sides, effectively eliminating the $y$ dimension. The voltage source on the input end had sinusodial variation in time and Gaussian variation in $x$. This had the effect of introducing a Gaussian beam with a wavelength of $1 \mu \mathrm{m}$ and an initial beam waist of $w_{0}=4 \mu \mathrm{m}$. Propagation was simulated through 2500 times steps of $\Delta t=1.0 \times 10^{-16} s$ with 1 st order basis functions and time steps, in a material with $\mu=\mu_{0}, \varepsilon=\varepsilon_{0}, \chi^{(3)}=2.0 \times 10^{-18}$, and $\tau=T=3.333333 \times 10^{-15} s$. Given those parameters, the Kelly formula yields a critical peak electric field value of $E_{c r}=7.625 \times 10^{7} \mathrm{~V} / \mathrm{m}$. Electric field magnitudes were set in terms of this critical value at $E_{0}=\frac{1}{4} E_{c r}, E_{0}=E_{c r}, E_{0}=\frac{3}{2} E_{c r}$, and $E_{0}=$ $3 E_{c r}$ in 4 different simulations. Finally, at the end of the simulation the sinusodial time variation was removed from the $\mathbf{E}$ field, allowing the creation of the contour plots found in Figure 3.

These plots yield results that are in good agreement with those obtained by the Kelly formula (26). The beam with power below critical is clearly diverging and both beams with powers above critical are clearly self-focusing. The beam at the estimated critical power is slowly diverging indicating that the simulated critical power is very near the power estimated by (26). The small discrepacd fisncy is accounted for by the induced conductivity that arises from $\dot{d}_{e f f}$. This conductivity introduces a small amount of attenuation in the waves that the Kelly formula does not model.

\section{Nonlinear Waveguides}

Metallic waveguides filled with $\chi^{(3)}$ nonlinear materials display some interesting nonlinear effects. As is the case with linear waveguides, the fields of such a guide can be decomposed in a series of Transverse Electric (TE) and Transverse Magnetic (TM) modes. However, in nonlinear waveguides power is allowed to couple 

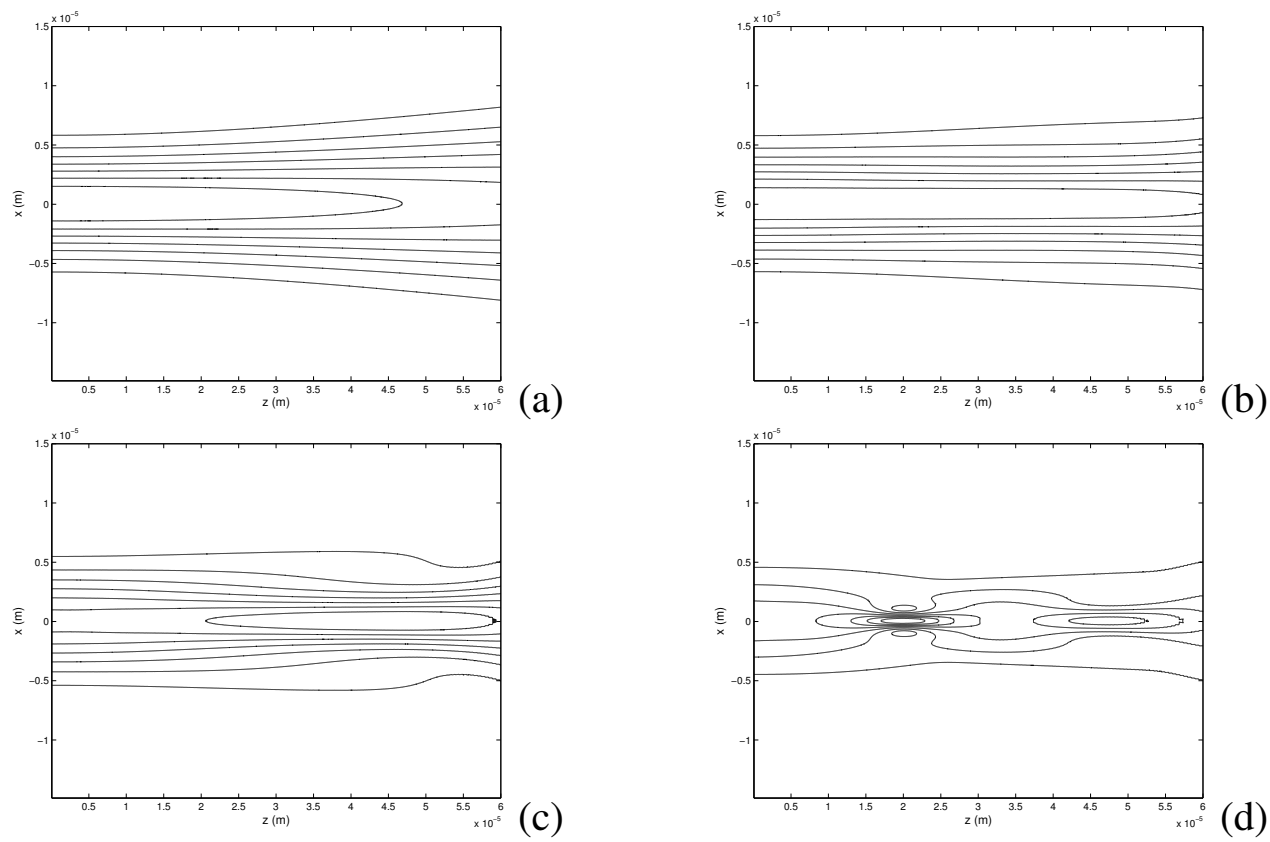

(b)

Fig. 3. Contour plots of simulated Gaussian beam magnitudes with initial magnitudes (a) $E_{0}=\frac{1}{4} E_{c r}$, (b) $E_{0}=E_{c r}$, (c) $E_{0}=\frac{3}{2} E_{c r}$, and (d) $E_{0}=3 E_{c r}$, where $E_{c r}$ is the critical peak electric field value predicted by the Kelly formula.

from one mode to another. Some analytical work has been done in the past to describe simple coupling between the two lowest order modes [31]. Recent analysis has considered the more general case of exicitation of many modes by a dominant fundamental mode [32]. However, when the minor mode powers become a significant fraction of the dominant mode power all the modes begin to interact in a far more complicated manner.

As a display of capability for the method, we simulated general waveguide mode mixing inside guides that were rectangular and circular in cross-section. The rectangular guide was $0.2 m \times 0.4 m \times 1 \mathrm{~mm}$ modeled with an $80 \times 1 \times 2000$ block of 1 st order elements. The material parameters were that of a dielectric glass with $n_{0}=1.5$ and $n_{2}=6.66666 \times 10^{-19}$ with the outer walls given PEC boundary conditions. The input end of the waveguide was excited with the TE20 mode of the guide using a wavelength of $\lambda=20 \mu \mathrm{m}$ and a field strength of $5 \times 10^{8} \mathrm{~V} / \mathrm{m}$. The simulation was run for 4000 time steps of $\Delta t=1.25 \times 10^{-15} s$ and the $y$ component of the $\mathbf{E}$ field data was captured at the final time. By computing Fourier transforms across $x$ in the guide, the magnitude in each mode was found at various locations in $z$ (see Figure 4). This provides a view into the power transfer occuring between the modes. At the input end of the guide the all of the power is in the TE20 mode that is being excited by the boundary condition. As the waves begin to travel in $z$, power is rapidly drained from the TE20 mode and transfered into higher modes. Once there is enough power in the higher modes they begin to couple to one another and even transfer power back to the TE20 mode. 

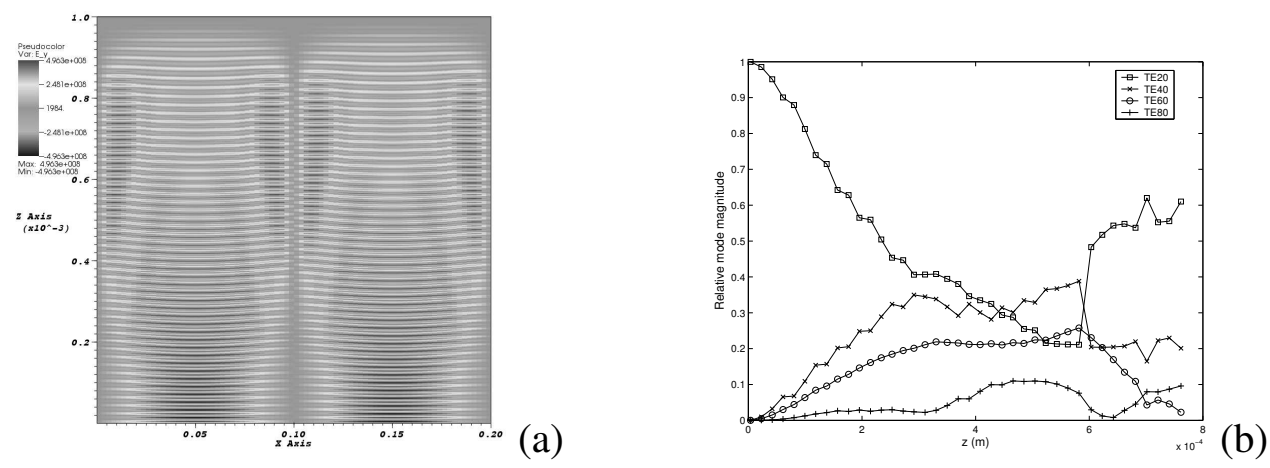

Fig. 4. (a) The $y$ component of the $\mathbf{E}$ field at $t=5 \mathrm{ps}$. The introduction of higher order modes can be seen clearly toward the end of the guide. (b) Magnitudes found in the first four even modes normalized to their sum.

The circular guide was a $150 \mu m$ long cylinder of radius $0.25 \mathrm{~m}$ modeled with an 180 element butterfly cross section in $x y$ (see Figure 5) and 500 elements in the propagation direction $z$. The input end was excited with the TE11 mode of the guide with a wavelength of $1.5 \mu \mathrm{m}$ and an $\mathbf{E}$ field strength of $1.6 \times 10^{9} \mathrm{~V} / \mathrm{m}$ at the center of the mode. The guide was filled with a dielectric glass with $n_{0}=1.5$ and $n_{2}=6.66666 \times 10^{-19}$ and a PEC boundary condition was applied to the outer wall. The simulation was run for 2500 time steps of $\Delta t=3 \times 10^{-16} \mathrm{~s}$. The $2 \mathrm{nd}$ order basis functions were used for the $\mathbf{E}$ and $\mathbf{B}$, the 1st order basis functions were used for the $\chi^{N L}$ field, and the 2 nd order time integration scheme was used to obtain improved accuracy.

Using these parameters the simulation required 2, 210, 464 1-form DOFs, 2, 184, 720 2-form DOFs, and 90,000 3-form DOFs. The matrices were decomposed using (17) and shared a 1-form $P$ matrix with 4,860,000 non-zero entries, a 2-form $P$ matrix with 3, 240,000 non-zero entries, and a 3-form $P$ matrix with 90,000 non-zero entries. The simulation required $4 G$ matrices, 2 with $54 \times 54$ element blocks, and 2 with $36 \times 54$ element blocks. However, these blocks were only stored for 145 geometrically distinct elements yielding a total storage requirement for $G$ matrices of 1,409, 400 real values. The computation time involved in taking a time step was dominated by the linear solve involving $M_{d_{e f f}}$. We used the Preconditioned Conjugate Gradient (PCG) method with a diagonal preconditioner to handle this solve. On average, the PCG method converged to a tolerance of $1 \times 10^{-21}$ in 8 iterations. While this method is more computationally expensive than a full wave FDTD approach, the results are far more accurate. This improved accuracy comes from a more geometrically accurate mesh that avoids FDTD "stairstepping" problems, and higher order basis functions that yield vastly improved dispersion error.

At the end of the simulation $\mathbf{E}$ field values were captured at a series of cross sections orthogonal to $z$ throughout the guide. In order to measure the distribution of power in the waveguide modes at each location, a least squares analysis was performed. This analysis fit the cross-sectional data to a model that consisted of a block of 16 modes ranging from TE10 to TE43 allowing us to examine the power transfer 


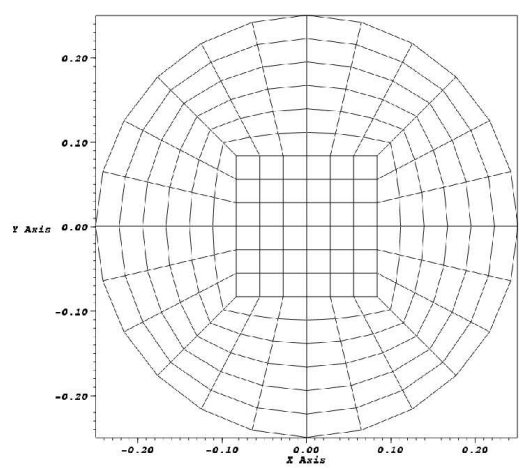

(a)
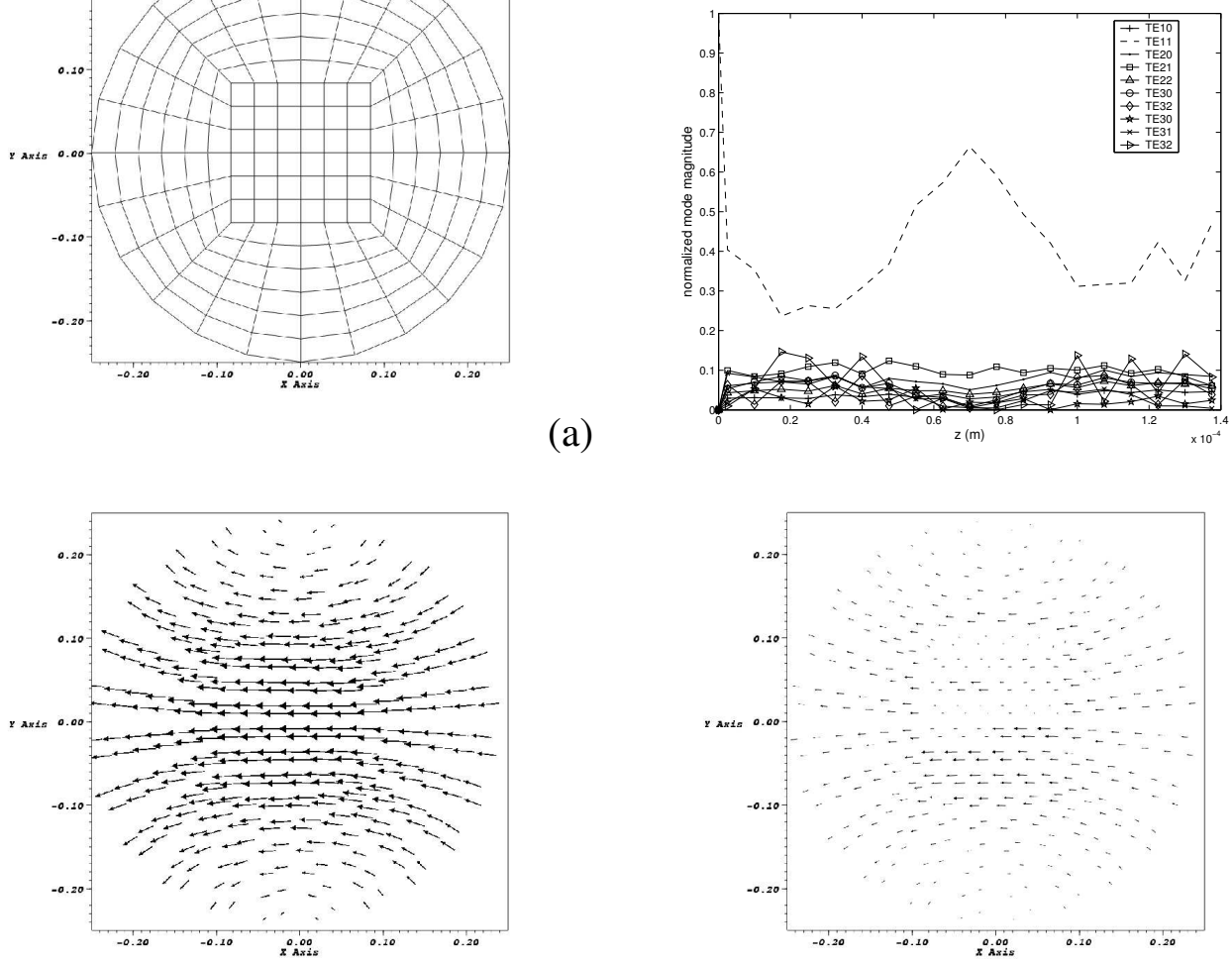

(c)

(d)

Fig. 5. (a) 180 element butterfly pattern used for the cross section of the circular waveguide mesh. (b) Normalized magnitudes found in the modes of the circular guide at various locations in $z$ (only modes with significant magnitude are displayed). (c) Plot of the initial TE11 E field. (d) Plot of the $\mathbf{E}$ field after significant mode mixing (at $z=1.375 \times 10^{-4} \mathrm{~m}$ in the guide).

occuring between those modes (see Figure 5).

After the the TE11 mode is injected into the guide, power is rapidly transfered into a variety of other modes, with the TE21 and TE32 modes receiving the most power. As was the case with the rectangular guide, the higher order modes begin to transfer power back into the original TE11 mode. After the TE11 mode gains enough power, it begins to transfer it back to the other modes setting up an oscillatory behavior. This behavior is similar to that found in the simpler case where power is only allowed to couple between 2 low order modes [31].

\section{Conclusions}

We have presented a mixed VFEM designed to model electromagnetics with a nonlinear polarization term on complicated geometries. The method uses arbitrary or- 
der Nedelec basis functions within a framework of discrete differential forms to model the fields in Maxwell's equations. Additionally, a series of optimizations that extends the range of problems that can be simulated with this method was discussed. The method has levels of dispersion error similar to linear VFEM methods, and produces resonable results for nonlinear optics test problems, making it suitable for use in computational nonlinear electromagnetics.

\section{References}

[1] J. D. Joannopoulos, R. D. MEade, J. N. Winn, Photonic Crystals: Molding the Flow of Light, Princeton University Press, Princeton, New Jersey, 1995.

[2] B. E. Little, S. T. Chu, W. Pan, Y. Kokubun, Microring resonator arrays for vlsi photonics, IEEE Photonics Tech. Lett. 12 (3) (2000) 323-325.

[3] R. R. Alfano, The supercontinuum laser source, Springer Verlag, 1989.

[4] R. Boyd, Nonlinear Optics, Academic Press, 2003.

[5] T. Fujisawa, M. Koshiba, Time-domain beam propagation method for nonlinear optical propagation analysis and its application to photonic crystal circuits, J. of Lightwave Tech. 22 (2) (2004) 684-691.

[6] R. M. Joseph, A. Taflove, Fdtd maxwell's equations models for nonlinear electrodynamics and optics, IEEE Trans. Ant. Prop. 45 (3) (1997) 364-374.

[7] R. Ziolkowski, Full-wave vector maxwell equation modeling of the self-focusing of ultrashort optical pulses in a nonlinear kerr medium exhibiting a finite response time, J. Opt. Soc. Am. B 10 (2) (1993) 186-198.

[8] J. C. Nédélec, Mixed finite elements in R3, Numer. Math. 35 (1980) 315-341.

[9] A. Bossavit, Whitney forms: a class of finite elements for three-dimensional computations in electromagnetism, IEEE Proceedings. 135 (8) (1988) 493-500.

[10] A.

Bossavit,

Computational Electromagnetism: Variational Formulation, Complementarity, Edge Elements., Academic Press, 1998.

[11] A. Bossavit, Generalized finite differences in computational electromagnetics, Progress in Electromagnetics Research 32 (2001) 45-64.

[12] P. Monk, Analysis of finite element method for Maxwell's equations 63 (1) (1992) 243-261.

[13] P. Monk, A finite element method for approximating the time-harmonic maxwell equations, SIAM J. Num. Anal. 28 (6).

[14] P. Monk, An analysis of Nédélec's method for the spatial discretization of Maxwell's equations, J. Comput. Appl. Math. 47 (1993) 101-121. 
[15] P. Monk, Finite element methods for Maxwell's equations, first edition Edition, Numerical Analysis and Scientific Computing, Oxford University Press, 2003.

[16] R. Hiptmair, Canonical construction of finite elements, Math. Comp. 68 (228) (1999) $1325-1346$.

[17] R. Hiptmair, Discrete Hodge operators: An algebraic perspective, J. Electromagnteic Waves Appl. 15 (3) (2001) 343-344.

[18] P. B. A. Deraemaeker, I. Babuska, Dispersion error and pollution of the FEM solution for the Helmholtz equation in one, two, and three dimensions 46 (4) (1999) 471-499.

[19] D. A. White, Numerical dispersion of a vector finite element method on skewed hexahedral grids, Commun. Numer. Meth. Engng. 16 (2000) 47-55.

[20] G. Rodrigue, D. White, A vector finite element time-domain method for solving maxwell's equations on unstructured hexahedral grids, SIAM J. Sci. Comp. 23 (3) (2001) 683-706.

[21] M. Ainsworth, Dispersive properties of high-order Nedelec/edge element approximation of the time-harmonic Maxwell equations, Philisophical Transactions of the Royal Society of London 362 (1816) (2004) 471-491.

[22] R. Rieben, D. White, G. Rodrigue, High order symplectic integration methods for finite element solutions to time dependent maxwell equations, IEEE Trans. Ant. Prop.In press.

[23] R. Rieben, D. White, G. Rodrigue, A high order mixed vector finite element method for solving the time dependent Maxwell equations on unstructured grids, J. Comput. Phys. 204 (2005) 490-519.

[24] A. Fisher, R. N. Rieben, G. H. Rodrigue, D. A. White, A generalized mass lumping technique for vector finite element solutions of the time-dependent maxwell equations, IEEE Trans. Ant. Prop. 53 (9) (2005) 2900-2910.

[25] J. D. Jackson, Classical Electrodynamics, 1962.

[26] P. Castillo, J. Koning, R. Rieben, D. White, A discrete differential forms framework for computational electromagnetics, Computer Modeling in Engineering \& Sciences 5 (4) (2004) 331-346.

[27] S. Vavasis, Stable finite elements for problems with wild coeffecients, SIAM Journal on Numerical Analysis 33 (3) (1996) 890-916.

[28] R. Rieben, D. White, Verification of high-order mixed fem solution of transient magnetic diffusion problems, IEEE Trans. Mag.Article in press.

[29] S. A. Akhmanov, R. V. Khokhlov, A. P. Sukhorukov, Self-focusing, self-defocusing, and self-modulation of laser beams, in: Laser Handbook, Vol. 2.

[30] P. L. Kelley, Self focusing of optical beams, Physical Review Lett. 15 (26) (1965) 1005-1008. 
[31] Y. Silberberg, G. I. Stegeman, Nonlinear coupling of waveguide modes, Appl. Phys. Lett. 50 (13) (1987) 801-803.

[32] D. Sjoberg, Nonlinear waveguides, Radio Science 38 (2) (2003) VIC 20-1.

This work was performed under the auspices of the U. S. Department of Energy by University of California, Lawrence Livermore National Laboratory under Contract W-7405-Eng-48. 